\title{
Hinweise fíir Autoren
}

Die nachstehenden Richtlinien sollen dazu dienen, den Autoren bei der Vorbereitung ihrer Publikationen zu helfen. Durch genaues Be-folgen können nachträgliche Korrekturen und damit verbundene Mühe sowie entsprechende Kosten vermieden werden.

Manuskripte in deutscher oder englischer Sprache sollen eine Zeilen-länge von 60 Anschlägen (Zwischenräume mitgerechnet) haben und mit doppeltem Zeilenabstand geschrieben werden. Die Manuskript-seite soil 30 Zeilen nicht überschreiten. Vier Manuskriptseiten ent-sprechen dann etwa einer Druckseite Normaltext. Für kurze wissen-schaftliche Mitteilungen beträgt das Umfangslimit IV2 Druckseiten einschließlich einer Tabelle oder Abbildung (1 Druckseite Kleindruck entspricht 4V2 Manuskriptseiten mit je 20 Zeilen zu 60 Anschlägen). DasManuskript soil in druckreifemZustand auf nicht durch-scheinendem uormalformatigem Schreibmaschinenpapier in doppel-ter Ausfertigung eingereicht werden. Die Entscheidung über die Auf-nahme erfolgt aufgrund mehrerer Gutachten. Eine Kopie des Manuskrip $1 \frac{1}{8}$ soil grundsätzlich beim Autor verbleiben. Die Korrektur der Druckfahnen ist Aufgabe der Autoren.

Der Autor erhält pro Druck- bzw. Textseite (Abbildungen werden nicht honoriert) ein Honorar von DM 60,-. Mit der Zahlung dieses Honorars erwirbt der Verlag alle Rechte an Sonder- und Abdrucken in anderen Publikationen. Autorkorrekturen über 10\% hat der Autor zu tragen, ebenso gehen Bildumzeichnungskosten zu seinen Lasten, falls er keine einwandfreien Vorlagen stellt. Dem Autor stehen 25 Gratissonderdrucke seiner Arbeit zu.

Schreibweise und Korrekturzeichen richten sich im Prinzip nach dem Duden. Bei Fachwörtern kann man sich weitgehend nach dem Pschyrembel richten, der eine vernünftige c-k-z-Schreibung anwendet.

Markennamen müssen, wenn sie geschützt sind, mit einem hochge-stellten ${ }^{\circledR}$ bezeichnet werden. Die Verantwortung für die korrekte Befolgung dieser Vorschrift liegt ausschließlich beim Autor, der Verlag lehnt jede Verantwortung ab.

Abkürzungen sollen möglichst sparsam verwendet werden. Ohne weiteres dürfen abgekürzt werden:

usw. $=$ und so weiter (nicht etc.)

vgl. $=$ vergleiche

bzw. $=$ beziehungsweise

evtl. = eventuell (nicht ev.!)

z. B. = zumBeispiel

Ferner steht es den Autoren natürlich frei, für häufig auftretende Ausdrücke im Sinne einer Platzersparnis spezielle Abkürzungen einzuführen. Der Autor sollte aber jeweils beim ersten Auftreten das Wort ausschreiben und die später verwendete Abkürzung in Klammern folgen lassen: z. B. Adenosin-monophosphat (AMP). 
Der Titel der Arbeit ist durch Unterstreichung hervorzuheben und von Untertiteln gut zu unterscheiden. Ein Kurztitel von nicht mehr als 60 Buchstaben muß alien Arbeiten beigefügt werden.

Institutsangaben gehören zu jeder Arbeit. Auf der ersten Seite ist in der Sprache, in der die Arbeit abgefaßt ist, das Institut anzugeben, aus dem die Arbeit hervorgegangen ist.

Zusammenfassungen sollen 10 Schreibmaschinenzeilen nicht überschreiten. Eine Fassung in englischer Sprache und der englische Titel der Arbeit müssen mitgeliefert werden, auch wenn die Arbeit in deutscher oder französischer Sprache abgefaßt ist. In die Zusam-menfassung gehören keine Literaturzitate, Tabellen, Abbildungen und Abbildungshinweise.

Key Words sollen ebenfalls in deutscher und englischer Sprache abgefaßt werden, auch wenn die Arbeit in deutscher oder französischer Sprache abgefaßt ist. Zu einer Arbeit gehören drei bis fünf Key Words (Schlüsselwörter).

Eigennamen sind in Verbindung mit einer zitierten Literatur im Text mit Großbuchstaben zu schreiben: MÜLLER (1973) und NEU-HAUS (1972). Von drei und mehr Autoren an wird nur noch der erste Name, mit dem Zusatz et al. geschrieben: PENN et al. (1971). Eigennamen in Verbindung mit Färbemethoden, Operationsmetho-den, Verfahren, Instrumenten, körpertopographischen Begriffen usw. werden nicht in Großbuchstaben geschrieben. Auszeichnungen einzelner Wörter oder ganzer Sätze erfolgen durch Kursivsatz, VERSALSATZ oder gesperrter Satz werden in dieser Zeitschrift nicht angewendet. Worte oder Sätze, die ausgezeich-net werden sollen, sind zu unterstreichen.

Fußnoten werden im Text mit hochgestellten arabischen Ziffern (x 23 ) gekennzeichnet und unten auf die entsprechende Manuskript-seite geschrieben. Literaturhinweise gehören nicht in Fußnoten, son-dern in das Literaturverzeichnis.

Tabellen werden mit römischen Ziffern numeriert und müssen einen Titel haben. Sie sollen als Teil für sich zusammengefaßt am Schluß der Arbeit beigelegt werden. Im Text sind die Stellen anzugeben, wo die Tabellen einzuschalten sind.

Abbildungen werden arabisch numeriert und benötigen unbedingt Legenden, die auf einem separaten Blatt am Schluß der Arbeit bei-zufügen sind. Im Text ist die Stelle zu bezeichnen, wo die Abbildung einzuschalten ist. Als Vorlage für Klischees genügen in der Re-gel nur saubere Originalzeichnungen oder Photographien. Schrift auf Abbildungsvorlagen darf bei Verkleinerung der Zeichnung auf Spal-tenbreite nicht kleiner als 6 Punkt (etwa 2,5 mm) sein. Abbildungsvorlagen dürfen nicht aufgeklebt, gelocht oder mit Büroklammern angeheftet eingereicht werden und sollen auf der Rückseite den Namen des Autors und des Beitrages sowie die Bildnummer tragen. Ist eine exakte Beschriftung nicht möglich, wird sie vom Verlag in der auf den Reproduktionsmaßstab abgestimmten Größe ausgeführt. In diesem Fall dürfen die Hinweise dafür keinesfalls in der Abbildung selbst, sondern auf einem darüberliegenden transparenten Deckblatt angegeben werden. Das gilt besonders für Röntgenaufnahmen. Dort sind auch Abstriche oder gewünschte Bildausschnitte zu bezeichnen. Hinweispfeile oder -linien sind ebenfalls auf dem Deckblatt anzu-bringen, wobei die Endpunkte durch Einstich mit einer feinen Nadel auf der darunterliegenden Originalvorlage fixiert werden sollen. Es empfiehlt sich ferner, auf dem Deckblatt wichtige Abbildungspartien zu kennzeichnen, damit die Kunstanstalt bei der Ätzung ihr beson-deres Augenmerk darauf richten kann. Teilbilder sollen möglichst zum Tableau zusammengestellt werden; hierbei ist der Satzspiegel von $168 \times 230 \mathrm{~mm} z u$ berücksichtigen.

Adresse des Autors ist am Schluß der Arbeit vollständig anzugeben. Literaturverzeichnis. Es soil nur die im Text zitierten Publikationen, diese dafür lückenlos, enthalten. Das 
Literaturverzeichnis ist grundsätzlich alphabetisch zu ordnen. Bei umfangreicheren Literaturverzeichnissen ist aus Gründen der Platzersparnis und Textübersicht-lichkeit eine fortlaufende Numerierung erwünscht, welche gleich-zeitig zur Zitierung im Text verwendet werden soil.

a) Zeitschriftenbeiträge: 1. Sämtliche Autorennamen, gefolgt von den

Initialen der Vornamen. 2. Vollständiger Titel der Arbeit. 3. Abge-

kürzter Titel der Zeitschrift. 4. Bandnummer der Zeitschrift (in arabi

schen Ziffern, einmal unterstrichen, gefolgt von Doppelpunkt).

5. Erste und letzte Seitenzahl der Arbeit. 6. Erscheinungsjahr (in

Klammern), gefolgt von Punkt.

Beispiel:

Bauer, A. W.; Kirby, W. M. M.; Sherris, J. C, and Turck, M., jr.: Antibiotic susceptibility testing by a standardized single-dose method. Amer. J. clin. Path. 45:493-496 (1966).

b) Bücher: 1. Sämtliche Autorennamen, gefolgt von den Initialen der Vornamen. 2. Vollständiger Buchtitel. 3. Eventuell Auflage.

4. Eventuell erste und letzte Seitenzahl der zitierten Stelle. 5. Ver

lag, Erscheinungsort und Erscheinungsjahr in Klammern, gefolgt von

Punkt.

Beispiel:

Lucien, M.; Parisot, J. et Richard, G: Traité d'endocrinologie

(Doin, Paris 1925).

c) Beiträge aus Hand- und Lehrbüchern, Sammelbänden und Kongreßberichten: 1. Sämtliche Autorennamen, gefolgt von den Initialen der Vornamen. 2. Vollständiger Titel der Arbeit. 3. Namen der Bandherausgeber ohne Vornamen. 4. Titel des Bandes. 5. Eventuell Band nummer und Auflage. 6. Erste und letzte Seitenzahl des zitierten Beitrages. 7. Verlag, Erscheinungsort und Erscheinungsjahr in Klam mern, gefolgt von Punkt.

Beispiel:

Sharpless, S. K.: Hypnotics and sedatives. II. Miscellaneous agents; in Goodman and Gilman The pharmacological basis of therapeutics; 4th ed., pp. 121-134 (Macmillan, New York 1970). 